\title{
A SYNCHROPHASOR ASSISTED HYBRID STATE ESTIMATOR
}

\author{
Vedran Kirincic — Srdjan Skok — Dubravko Frankovic *
}

\begin{abstract}
The paper presents a Synchrophasor Assisted Hybrid State Estimator that utilizes the conventional SCADA measurements and the synchrophasors obtained from Phasor Measurement Units (PMUs). To take advantage of the high sampling frequency of the multiple sets of synchrophasors they are preprocessed in a recursive algorithm that provides the state estimate for the power system part observable by PMUs. The results are forwarded to an iterative procedure in which they are combined with SCADA measurements. The given solution was applied on the IEEE test systems with 14, 30 and 57 buses and its performance was compared with other state estimators. The filtering of measurement errors and convergence, while providing improved accuracy of the final state estimates of the developed methodology can be compared with other hybrid state estimators.

K e y w ords: hybrid state estimator, phasor measurement unit (PMU), power system state estimation, synchronized measurement technology
\end{abstract}

\section{INTRODUCTION}

The contemporary electric power system presents one of the most complex technical systems. With undergoing changes such as renewable sources integration and the electric energy market development, the operation of the power system becomes tighter and closer to its stability limits, with reduced security margins. Transmission System Operators (TSOs) worldwide are trying to cope with aging infrastructures, since the lack of resources and strict policies are restraining factors that are preventing them from building additional transmission assets. Emerging measurement technologies as well as advances in computational algorithms and a telecommunication infrastructure have contributed to new research and development applicable in the field of power systems. Providing the availability of voltage and current phasors from Phasor Measurement Units (PMUs) that are deployed across wide area, one obtains a real-time overview of the power system. Furthermore, the advanced applications include various solutions for Wide Area Monitoring, Protection and Control (WAMPAC) systems as well as special protection schemes for preserving the power system integrity and stability $[1,2]$.

One of the first applications that can significantly benefit from the high accuracy and the precise time stamps of synchrophasors is the power system state estimator. The state estimator is the cornerstone application that provides inputs to all subsequent applications such as the power flow calculation and the contingency analysis. The field of state estimation is still one of the hottest research topics $[3,4]$. Although TSOs are gradually deploying PMUs in their transmission systems, in the upcoming years power systems would still not become completely observable using only synchrophasors, which would eventually result in a linear state estimation. Considering the expenses associated with equipment and the engineering costs, as well as the availability of highly redundant conventional measurements, the efforts to optimally integrate synchrophasors into hybrid state estimators are justified [5].

Taking both the synchrophasors and the conventional SCADA measurements, the hybrid solutions seek for optimal performances regarding the estimation accuracy, filtering capabilities, convergence and computational speed [6]. An overview of literature reveals that there are many hybrid models proposed, such as the one that takes the rectangular format of the phasors of current into a measurement vector in order to mitigate convergence issues [7]. On the other hand, in order to avoid the propagation of uncertainties, in the estimator proposed in [8] the measurements are used directly without any transformation, but they are subjected to a set of constraints. The estimator that obtains pseudovoltages using the voltage and current phasors as well as the parameters of branches is given in [9]. Some other approaches take pseudo-flows and pseudo-injections into the measurement vector together with the voltage phasors and the SCADA measurements $[11,12]$. Since the optimization methods for optimal PMU placement have already been extensively investigated and are out of scope of this paper, an interested reader is suggested to refer to [13], in which a thorough description of the state of the art is provided.

In this paper a synchrophasor assisted hybrid state estimator (SAHSE) which first preprocesses the voltage and current phasors to provide the pseudo-measurements for a part of the system observable by the PMUs is proposed. After the preprocessing, an iterative procedure is applied, in which the obtained pseudo-measurements are merged with the conventional SCADA measurements

*Faculty of Engineering, University of Rijeka, Vukovarska 58, 51000 Rijeka, Croatia, vedran.kirincic@riteh.hr, srdjan.skok@riteh.hr, dubravko.frankovic@riteh.hr 


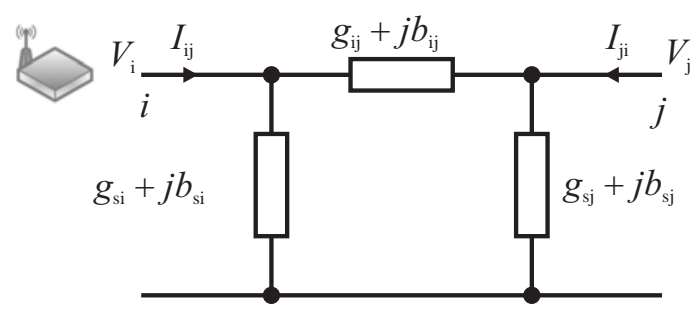

Fig. 1. A pi-model of the network branch

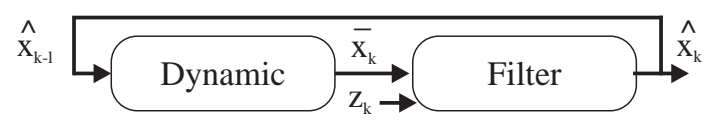

Fig. 2. The recursive filter structure

into the measurement vector and the state of the complete power system is estimated. The high sampling frequency at which the synchrophasors arrive at the control center opens the possibility to obtain the more accurate pseudo-measurements by using the consecutive sets of the synchrophasors that are used separately from the SCADA measurements. The proposed synchrophasor assisted state estimator was applied to three IEEE test systems, with 14, 30 and 57 buses. The simulated case studies assumed a slow change of the power system state in a minute range. The obtained results were compared with the results of the classical state estimator that uses the SCADA measurements, as well as with the results of other hybrid state estimators.

The theoretical background of the classical state estimation is given in Section 2, while Section 3 presents the proposed hybrid state estimator. In Sections 4 and 5 the case studies and the results are given, respectively. Section 6 concludes the paper.

\section{CLASSICAL APPROACH TO POWER SYSTEM STATE ESTIMATION}

Being the basis for the classical state estimators running worldwide, the Weighted Least Squares (WLS) method is briefly explained, since it is used as a part of the proposed hybrid state estimator.

The measurement vector $\mathrm{z}$ usually includes voltage magnitudes, active and reactive power flows and injections while the state vector $\mathrm{x}$ constitutes of a set of voltage angles and magnitudes. These two vectors are related using the set of nonlinear equations $\mathbf{h}(\mathbf{x})$ and the error vector $\boldsymbol{e}$ that contains uncorrelated measurement errors:

$$
\boldsymbol{z}=\boldsymbol{h}(\mathbf{x})+\boldsymbol{e}
$$

In order to differentiate the measurements according to their accuracy, the squares of the inverse of the standard deviations $\sigma$ are used as measurement weights. The standard uncertainties in the measurements can be obtained from the specified maximum uncertainties if one assumes a uniform probability distribution over the entire range of uncertainty $[14,15]$ :

$$
\sigma_{i}=\frac{\Delta u_{i}}{\sqrt{3}}
$$

where $\Delta u_{i}$ is the maximum uncertainty in the $i$-th measurement. The measurement error covariance matrix is formed as $\mathbf{R}=\operatorname{diag}\left\{\sigma_{1}^{2}, \ldots, \sigma_{m}^{2}\right\}$, where $m$ is a number of measurements. Satisfying the condition $\partial J(\mathbf{x}) / \partial \mathbf{x}=$ 0 , the optimal estimation is obtained once that the objective function $J(\mathbf{x})$ is minimized:

$$
J(\mathbf{x})=\frac{1}{2}[\boldsymbol{z}-\boldsymbol{h}(\mathbf{x})]^{\top} \mathbf{R}^{-1}[\boldsymbol{z}-\boldsymbol{h}(\mathbf{x})] .
$$

An iterative algorithm is applied since the relationship between the state vector elements and the conventional measurements is a nonlinear one. Introducing the gain matrix given as

$$
\mathbf{G}\left(\boldsymbol{x}^{k}\right)=\mathbf{H}^{\top}\left(\boldsymbol{x}^{k}\right) \mathbf{R}^{-1} \mathbf{H}\left(\mathbf{x}^{k}\right)
$$

and the Jacobian matrix

$$
\mathbf{H}=\partial \mathbf{h}(\mathbf{x}) / \partial \mathbf{x}
$$

the change of the state vector elements is calculated at the $k$-th iteration

$$
\begin{aligned}
\mathbf{G}\left(\mathbf{x}^{k}\right) \Delta \boldsymbol{x}^{k} & =\mathbf{H}^{\top}\left(\mathbf{x}^{k}\right) \mathbf{R}^{-1}\left[\boldsymbol{z}-\boldsymbol{h}\left(\mathbf{x}^{k}\right)\right], \\
\Delta \mathbf{x}^{k} & =\mathbf{x}^{k+1}-\boldsymbol{x}^{k} .
\end{aligned}
$$

The calculation stops when the value of $\Delta \boldsymbol{x}^{k}$ becomes smaller than the desired tolerance.

The ill-conditioning of the gain matrix, due to the highly accurate measurements such as zero-injections and consequently theirs high weights, is addressed in [3]. The introduction of zero-injections through a set of constraints helps to alleviate the issue:

$$
\begin{aligned}
& L(\mathbf{x}, \boldsymbol{\lambda})=\frac{1}{2}[\boldsymbol{z}-\boldsymbol{h}(\mathbf{x})]^{\top} \mathbf{R}^{-1}[\boldsymbol{z}-\boldsymbol{h}(\mathbf{x})]-\boldsymbol{\lambda}^{\top} \boldsymbol{c}(\mathbf{x}) \\
& \text { subject to } \boldsymbol{c}(\mathbf{x})=\mathbf{0} .
\end{aligned}
$$

Again, the first-order optimality conditions $\partial L(\boldsymbol{x}, \boldsymbol{\lambda}) / \partial \mathbf{x}=0$ and $\partial L(\boldsymbol{x}, \boldsymbol{\lambda}) / \partial \boldsymbol{\lambda}=0$, where $\boldsymbol{\lambda}$ is the vector of the Lagrange multipliers, need to be satisfied in order to minimize $L(\boldsymbol{x}, \boldsymbol{\lambda})$. With $\mathbf{C}=\partial \boldsymbol{c}(\mathbf{x}) / \partial \mathbf{x}$ and $\Delta \mathbf{x}=\mathbf{x}^{k+1}-\mathbf{x}^{k}$, the system of equations is iteratively solved for $\Delta \boldsymbol{x}$ and $\boldsymbol{\lambda}$ by using the Gauss-Newton method:

$$
\left[\begin{array}{cr}
\alpha \mathbf{H}^{\top} \mathbf{R}^{-1} \mathbf{H} & \mathbf{C}^{\top} \\
\mathbf{C} & \mathbf{0}
\end{array}\right]\left[\begin{array}{c}
\Delta \boldsymbol{x} \\
-\boldsymbol{\lambda}
\end{array}\right]=\left[\begin{array}{c}
\alpha \mathbf{H}^{\top} \mathbf{R}^{-1}\left(\boldsymbol{z}-\boldsymbol{h}\left(\boldsymbol{x}^{k}\right)\right) \\
-\boldsymbol{c}\left(\mathbf{x}^{k}\right)
\end{array}\right] .
$$

The gain matrix condition number is additionally minimized by using the scaling factor

$$
\alpha=\frac{1}{\max \left(\operatorname{diag}\left(\mathbf{R}^{-1}\right)\right)} .
$$




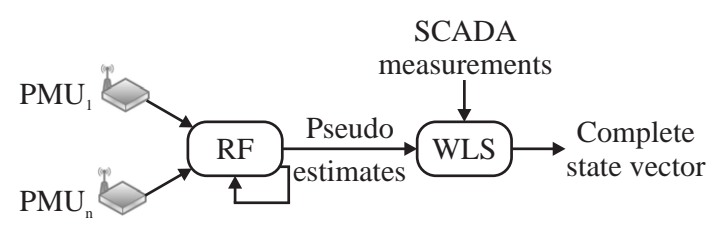

Fig. 3. The SAHSE architecture

Table 1. The elements of the Jacobian matrix for the current phasors

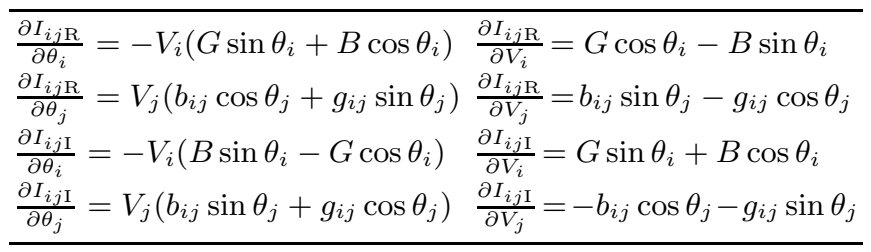

\section{SYNCHROPHASOR ASSISTED HYBRID STATE ESTIMATOR}

The proposed hybrid state estimator aims to utilize the high sampling frequency of synchrophasors in order to provide the more accurate pseudo-measurements, and finally the more accurate estimation of the complete state vector. Let us consider a pi-model of the network branch, as given in Fig. 1. Having the PMU deployed at bus $i$ and knowing the branch conductance $g$ and susceptance $b$, both buses become observable. The voltage phasor $\mathbf{V}_{i}$ at the bus $i$ and the current phasor $\mathbf{I}_{i j}$ between the buses are measured, while the voltage phasor $\mathbf{V}_{j}$ at the neighboring bus $j$ is calculated.

When more PMUs are deployed in the system, the sets of measured voltage and current phasors are combined into the measurement vector $\boldsymbol{z}_{\text {PreProc }}$ of the preprocessor:

$$
\boldsymbol{Z}_{\text {PreProc }}=\left[\begin{array}{c}
\mathbf{V}_{i \text { Polar }} \\
\boldsymbol{I}_{i j \text { Rect }}
\end{array}\right]=\left[\begin{array}{c}
{\left[\begin{array}{c}
\boldsymbol{\theta}_{i \text { Ang }} \\
\mathbf{V}_{i \text { Mag }}
\end{array}\right]} \\
{\left[\begin{array}{c}
\mathbf{I}_{i j \mathrm{R}} \\
\mathbf{I}_{i j \mathrm{I}}
\end{array}\right]}
\end{array}\right] \text {. }
$$

The voltage phasors measured at the PMU buses are taken in polar coordinates and are grouped into the vector $\boldsymbol{V}_{i \text { Polar }}$, where $\mathbf{I}_{i j \mathrm{R}}$ and $\mathbf{I}_{i j \text { Rect }}$ are the vectors of voltage magnitudes and angles, respectively. The measured current phasors are transformed from polar to rectangular coordinates and are grouped into the vector $\mathbf{I}_{i j \text { Rect }}$, where $\mathbf{I}_{i j \mathrm{R}}$ and $\mathbf{I}_{i j \mathrm{I}}$ are the vectors of real and imaginary parts, respectively. The transformation of each current phasor $\mathbf{I}$ from polar to rectangular coordinates is obtained as

$$
\mathbf{I}=I \angle \phi=i_{\mathrm{R}}+j i_{\mathrm{I}}=I \cos \phi+j I \sin \phi
$$

where $I$ and $\phi$ are the current magnitude and phase angle while $i_{\mathrm{R}}$ and $i_{\mathrm{I}}$ are the real and imaginary parts, respectively. Expressed as the functions of the state vector elements, the real and imaginary parts of the current phasors are given as

$$
\begin{gathered}
I_{i j \mathrm{R}}=V_{i} G \cos \theta_{i}-V_{i} B \sin \theta_{i}+b_{i j} V_{j} \sin \theta_{j}-g_{i j} V_{j} \cos \theta_{j} \\
I_{i j \mathrm{I}}=V_{i} B \cos \theta_{i}-V_{i} G \sin \theta_{i}-b_{i j} V_{j} \cos \theta_{j}-g_{i j} V_{j} \sin \theta_{j}
\end{gathered}
$$

where $G=g_{i j}+g_{s i}, B=b_{i j}+b_{s i}$.

The classical uncertainty propagation theory is applied for the calculation of uncertainty propagation [15]. The standard deviations of the real and imaginary parts are computed using the standard deviations of the magnitude and angle

$$
\begin{aligned}
\sigma_{i \mathrm{R}} & =\sqrt{\left(\frac{\partial i_{\mathrm{R}}}{\partial I}\right)^{2} \sigma_{\mathrm{I}}^{2}+\left(\frac{\partial i_{\mathrm{R}}}{\partial \phi}\right)^{2} \sigma_{\phi}^{2}} \\
& =\sqrt{\sigma_{\mathrm{I}}^{2} \cos ^{2} \phi+\sigma_{\phi}^{2}(I \sin \phi)^{2}} \\
\sigma_{i \mathrm{I}} & =\sqrt{\left(\frac{\partial i_{\mathrm{I}}}{\partial I}\right)^{2} \sigma_{\mathrm{I}}^{2}+\left(\frac{\partial i_{\mathrm{I}}}{\partial \phi}\right)^{2} \sigma_{\phi}^{2}} \\
& =\sqrt{\sigma_{\mathrm{I}}^{2} \sin ^{2} \phi+\sigma_{\phi}^{2}(I \cos \phi)^{2}}
\end{aligned}
$$

The state vector $\boldsymbol{x}_{\text {PreProc }}$ comprises the subset of voltage phasors $x_{\mathrm{PMU}}$ at PMU buses and the subset of voltage phasors $\boldsymbol{x}_{\text {Neighbor }}$ at neighboring buses:

$$
\mathbf{X}_{\text {PreProc }}=\left[\begin{array}{c}
\boldsymbol{X}_{\mathrm{PMU}} \\
\boldsymbol{x}_{\text {Neighbor }}
\end{array}\right] \text {. }
$$

Assuming the sufficient number of measurement channels to measure the voltage phasor at the PMU bus and the current phasors on coincident branches, each observable island consists of the PMU bus and its neighboring buses. The voltage phase angles $\theta_{i}$ at the $N$ buses in the island are indirectly connected and refer to a common angle reference $\theta_{0}$ of the slack bus

$$
\theta_{i}=\theta_{i}-\theta_{0}, \quad \forall i \in\{1, \ldots, N\}
$$

Instead of using a single set of synchrophasors, the several sets of consecutive PMU measurements are more likely to provide the more accurate state estimation. In order to take advantage of the high sampling rate of synchrophasors, a recursive filter is applied [16]. As given in Fig. 2, the system state at instant $k$ is obtained using the state estimate at instant $k-1$. The predicted state is then filtered and updated with the newest set of measurements. What follows, is a brief explanation of the procedure for the completeness of the paper.

The measurement model used in the paper is nonlinear due to the nonlinear relationship between the measurements of currents and the state vector elements. Assuming slow changes of loads in a minute range, the system state transition can be described using a linear discrete 
Table 2. Measurements locations for the IEEE 14 test system

\begin{tabular}{ll}
\hline \multicolumn{1}{c}{ Measurement type } & \multicolumn{1}{c}{ Measurement location } \\
\hline Voltage magnitude (\#bus) & $2,3,8,10,12$ \\
Power flow (\#from-\#to) & $1-2,4-7,4-9,5-6,6-12$, \\
& $6-13,7-9,13-14$ \\
Power injection (\#bus) & $1,2,3,4,6,8,9$, \\
Voltage phasor (\#bus) & $10,11,12,13,14$ \\
Current phasor (\#from-\#to) 1-2, 1-5, 6-5, 6-11, 6-12, 6-13 \\
\hline
\end{tabular}

Table 3. Measurements locations for the IEEE 14 test system

\begin{tabular}{ll}
\hline \multicolumn{1}{c}{ Measurement type } & \multicolumn{1}{c}{ Measurement location } \\
\hline Voltage magnitude (\#bus) & $2,3,6,9,11,14,16,17,25,30$ \\
& $1-3,2-4,2-6,4-6,5-7,6-8,6-9$, \\
& $6-10,12-13,12-15,14-15,16-17$, \\
Power flow (\#from-\#to) & $15-18,10-20,10-17,15-23,25-26$, \\
& $25-27,28-27,29-30,6-28$ \\
Power injection (\#bus) & $1,2,4,6,10,11,12,15$, \\
Voltage phasor (\#bus) & $18,19,24,25,27,30$ \\
& $1-2,1-3,7,4-6,5-7,15,16,17$ \\
Current phasor & $4-12,14-15,16-17,15-18,15-23$, \\
(\#from-\#to) & $4-2,4-3,5-2,7-5,7-6,9-6,14-12$, \\
& $15-12,16-12,15-14,17-16,17-10$ \\
\hline
\end{tabular}

Table 4. Measurements locations for the ieee 57 test system

\begin{tabular}{ll}
\hline \multicolumn{1}{c}{ Measurement type } & \multicolumn{1}{c}{ Measurement location } \\
\hline \multirow{2}{*}{ Voltage magnitude (\#bus) } & $1,3,4,5,7,8,11,15,17$, \\
& $22,27,31,37,44,52,54$ \\
& $1-15,1-17,2-3,3-4,4-5,4-18$, \\
& $6-7,7-8,7-29,8-9,9-10,9-11$, \\
Power flow & $9-12,9-13,12-16,12-17,13-15$, \\
(\#from-\#to) & $14-15,14-46,18-19,22-23,22-38$, \\
& $24-25,28-29,24-26,26-27,32-33$, \\
& $35-36,38-48,46-47,52-29,52-53$ \\
Power injection (\#bus) & $1,2,5,6,10,12,13,15,18$, \\
& $19,25,27,30,32,35,41,43$, \\
Voltage phasor (\#bus) & $14,47,49,51,53,54,55,57$ \\
& $14,15,16,18,11,13,41,42,57$ \\
& $1-2,3-4,8-9,13-14,13-15$, \\
& $1-15,1-16,1-17,3-15,5-6,10-12$, \\
Current phasor & $11-13,14-15,18-19,11-41,41-42$, \\
(\#from-\#to) & $41-43,15-45,14-46,10-51,13-49$, \\
& $11-43,57-56,3-2,5-4,8-6,10-9$, \\
& $11-9,13-9,14-13,15-13,15-1$, \\
& $16-1,15-3,18-4,18-4,8-7,13-11$, \\
& $13-12,16-12,15-14,41-11,42-41$, \\
& $41-56,42-56,57-39$ \\
\hline
\end{tabular}

time prediction model to capture the quasi steady-state behavior of the system

$$
\begin{aligned}
\boldsymbol{z}_{k} & =\boldsymbol{h}\left(\mathbf{x}_{k}+\mathbf{r}_{k},\right. \\
\mathbf{x}_{k} & =\mathbf{F}_{k-1} \mathbf{x}_{k-1}+\mathbf{g}_{k-1}+\boldsymbol{q}_{k-1} .
\end{aligned}
$$

The vectors $\boldsymbol{r}$ and $\boldsymbol{q}$ are the white Gaussian noise of the measurement and prediction models with the covari- ance matrices $\mathbf{R}$ and $\mathbf{Q}$, respectively. The relationship between the states at instants $k$ and $k-1$ is described by using the matrix $\mathbf{F}$ and the vector $\mathbf{g}$, which are updated using the Holt's method [17]

$$
\begin{aligned}
& \mathbf{F}_{k}=\alpha_{k}\left(1+\beta_{k}\right) \mathbf{I} \\
& \boldsymbol{g}_{k}=\left(1+\beta_{k}\right)\left(1-\alpha_{k}\right) \overline{\mathbf{x}}_{k}-\beta_{k} \mathbf{a}_{k-1}+\left(1-\beta_{k}\right) \boldsymbol{b}_{k-1} .
\end{aligned}
$$

where $\mathbf{I}$ is the identity matrix, while $\alpha_{k}$ and $\beta_{k}$ are constants in the range from 0 to 1 . The vectors $\vec{a}_{k}$ and $\vec{b}_{k}$ are obtained as

$$
\begin{aligned}
& \mathbf{a}_{k}=\alpha_{k} \hat{\boldsymbol{x}}_{k}+\left(1-\alpha_{k}\right) \overline{\mathbf{x}}_{k}, \\
& \boldsymbol{b}_{k}=\beta_{k}\left(\mathbf{a}_{k}-\mathbf{a}_{k-1}\right)+\left(1-\beta_{k}\right) \boldsymbol{b}_{k-1} .
\end{aligned}
$$

The state vector is estimated at instant $k$ as

$$
\hat{\mathbf{x}}_{k}=\overline{\mathbf{x}}+\mathbf{K}_{k}\left(\mathbf{z}_{k}-\mathbf{H}_{k} \overline{\mathbf{x}}_{k}\right) .
$$

where $\mathbf{H}_{k}$ is the Jacobian matrix and $\mathbf{K}_{k}$ is the pseudoinverse matrix given as

$$
\mathbf{K}_{k}=\mathbf{P}_{\overline{\mathbf{x}}_{k}} \mathbf{H}_{k}^{\top}\left(\mathbf{H}_{k} \mathbf{P}_{\overline{\mathbf{x}}_{k}} \mathbf{H}_{k}^{\top}+\mathbf{R}_{k}\right)^{-1}
$$

While the elements of the Jacobian matrix for the voltage phasors are straightforward, either 0 or 1 depending on PMU locations, the elements of the Jacobian matrix for the current phasors need to be derived using the expressions given in Tab. 1.

In each run of the preprocessor, during the period in which the SCADA measurements are collected, a new set of the synchrophasors is used. The state estimate from the last run is used as the pseudo-measurements and combined with the SCADA measurements in the WLS procedure which is described in Section 2. The measurement vector $\boldsymbol{z}_{\mathrm{WLS}}$ of the WLS estimator is

$$
\boldsymbol{Z}_{\mathrm{WLS}}=\left[\begin{array}{c}
\boldsymbol{z}_{\text {Pseudo }} \\
\boldsymbol{z}_{\text {SCADA }}
\end{array}\right]
$$

where $\boldsymbol{z}_{\text {Pseudo }}$ is the subset of pseudo-measurements comprising the estimated voltage magnitudes and angles at the buses observable by PMUs

$$
\boldsymbol{Z}_{\text {Pseudo }}=\hat{\mathbf{x}}_{\text {PreProc }}=\left[\begin{array}{ll}
\boldsymbol{\theta}_{\text {Pseudo }}, & \mathbf{V}_{\text {Pseudo }}
\end{array}\right]^{\top}
$$

and $\boldsymbol{z}_{\mathrm{SCADA}}$ is the subset of conventional measurements such as voltage magnitudes, active and reactive power flows and injections

$$
\mathbf{z}_{\mathrm{SCADA}}=\left[\mathbf{V}_{\text {mag }}, \mathbf{P}_{\text {flow }}, \mathbf{Q}_{\text {flow }}, \mathbf{P}_{i n j}, \mathbf{Q}_{i n j}\right]^{\top} .
$$

The state vector $\boldsymbol{x}_{\mathrm{WLS}}$ is the set of voltage phasors in polar coordinates at all system buses. Since the state vector estimated by the preprocessor $\hat{\boldsymbol{x}}_{\text {preProc }}$ is in polar 
Table 5. Maximum measurement uncertainties

\begin{tabular}{cccccc}
\hline Conventional measurements & \multicolumn{3}{|c}{ PMU measurements } \\
\hline $\begin{array}{c}\text { Voltage Power } \\
\text { magnitude flow }\end{array}$ & $\begin{array}{c}\text { Power } \\
\text { injection }\end{array}$ & $\begin{array}{c}\text { Current } \\
\text { magnitude magnitude }\end{array}$ & $\begin{array}{c}\text { Voltage } \\
\text { current } \\
\text { phase } \\
\text { angle }\end{array}$ \\
\hline $0.2 \%$ & $2 \%$ & $2 \%$ & $0.03 \%$ & $0.02 \%$ & $0.01^{\circ}$ \\
\hline
\end{tabular}

Table 6. The state estimators applied to the test systems

\begin{tabular}{llc}
\hline Abbreviation & \multicolumn{1}{c}{ Explanation } & Reference \\
\hline Classical & Classical state estimator & {$[3]$} \\
HCSE & Hybrid Constrained State Estimator & {$[8]$} \\
RectI & Rectangular Currents State Estimator & {$[7]$} \\
PsVolt & Pseudo Voltages State Estimator & {$[9]$} \\
PsFlow & Pseudo Flows State Estimator & {$[12]$} \\
PsMeas & Pseudo Measurements State Estimator & {$[10]$} \\
PsInj & Pseudo Injections State Estimator & {$[11]$} \\
\hline
\end{tabular}

coordinates, there is no transformation of measurements and the associated propagation of measurement uncertainties. The covariance matrix of the preprocessed states is used to obtain their weights for the WLS procedure

$$
\mathbf{P}_{\hat{\mathbf{x}}_{i}}=\left(\mathbf{I}-\mathbf{K}_{i} \mathbf{H}_{i}\right) \mathbf{P}_{\overline{\mathbf{x}}_{i}}
$$

where $\mathbf{I}$ is the identity matrix and $\mathbf{P}_{\overline{\mathbf{x}}_{i}}$ is the covariance matrix of the prediction error

$$
\mathbf{P}_{\overline{\mathbf{x}}_{i}}=\mathbf{F}_{i} \mathbf{P} \hat{\mathbf{x}}_{i-1} \mathbf{F}_{i}^{\top}+\mathbf{Q}_{i}
$$

In Fig. 3 the SAHSE architecture is given. The preprocessor runs a certain number of times and each time it takes the estimation (prediction) from its previous run, refreshes it with the latest set of synchrophasors and estimates the system state for the part of the system observable by the PMUs. The result of the last run is taken as a part of the measurement vector in the WLS state estimator together with the SCADA measurements.

In the proposed solution, the synchrophasors and the SCADA measurements are processed separately. The latter allows that in the cases of synchrophasors unavailability due to PMU outages or GPS failures, the state estimate can be obtained using the SCADA measurements only, which are highly redundant and make the majority of modern systems fully observable. As the number of the available synchrophasors gradually increases, the area observable by the PMUs becomes larger and the state estimator performance is improved.

\section{CASE STUDIES}

To test its performance, the developed hybrid state estimator was applied to three standard IEEE test systems of different sizes, topologies and configurations of measurements [18]. For the IEEE test systems with 14, 30 and 57 the SCADA measurements were placed for complete observability by running the observability analysis. Furthermore, the redundancy analysis was used to determine the locations of the PMUs in order to improve local redundancy levels. Tables $2-4$ provide the placement of the conventional measurements and the PMUs in the test systems.

The maximum measurement uncertainties are usually provided for the each type of measurement equipment and are used to derive the standard uncertainties by using expression $(2)[8,19,20]$. The power flow calculation output is taken as the set of true measurements, to which the random Gaussian noise with a zero mean and the given uncertainties was added to obtain the noisy measurements. The maximum measurement uncertainties are given in Tab. 5 .

In order to compare the developed hybrid model with other state estimators, the classical state estimator and several hybrid models were implemented and applied to the test systems. Table 6 gives the list of the implemented state estimators.

To unbias the results, a Monte Carlo analysis with 100 trials was carried out and the average result was taken. In each Monte Carlo trail, the sample for each measurement differed according to randomly created noise. The metric used to compare the accuracy of the state estimators was the variance of the estimated states $\sigma_{\Sigma}^{2}$ of the complete power system

$$
\sigma_{\sum}^{2}=\frac{1}{M} \sum_{i=1}^{M} \sum_{j=1}^{L}\left(\hat{\mathbf{x}}(j)_{(i)}-\mathbf{x}(j)\right)^{2}
$$

where $\hat{\boldsymbol{x}}_{(i)}$ is the estimated state vector in the $i$-th Monte Carlo trail, $\mathbf{x}$ is the state vector with true state values, $M$ is the number of the Monte Carlo trials carried out, $L$ is the number of the state variables in the power system.

The ability of the state estimator to filter measurement errors is expressed through the filtering index $\xi(\hat{x})$, in which the offset of the calculated values $\hat{z}$ and the noisy measurements $z$ from the true values $z^{\text {true }}$ is obtained as

$$
\xi(\hat{x})=\frac{1}{M} \sum_{i=1}^{M} \frac{\sum_{j=1}^{m}\left(\hat{z}_{j}-z_{j}^{\text {true }}\right)^{2}}{\sum_{j=1}^{m}\left(z_{j}-z_{j}^{\text {true }}\right)^{2}}
$$

where $M$ is the number of Monte Carlo trials and $m$ is the number of measurements.

To investigate the convergence and speed of the state estimators, the number of iterations and the computation time necessary to reach the convergence criterion of $10^{-6}$ were used. 
Table 7. State estimation results for the IEEE 14 test system

\begin{tabular}{cccccc}
\hline System & SE & $\sigma_{\Sigma}^{2}$ & $\xi$ & Time $[\mathrm{s}]$ & Iter. \\
\hline \multirow{6}{*}{14} & Classical & $2.2123 \times 10^{-3}$ & 0.8192 & 0.016 & 4.99 \\
& HCSE & $2.7961 \times 10^{-5}$ & 0.0926 & 0.025 & 5.00 \\
IEE & RectI & $2.7961 \times 10^{-5}$ & 0.0926 & 0.019 & 5.05 \\
& PsVolt & $2.7043 \times 10^{-5}$ & 0.0801 & 0.016 & 4.07 \\
& SAHSE & $2.6798 \times 10^{-5}$ & 0.0835 & $0.022+0.012$ & $1+2.98$ \\
& PsFlow & $2.7955 \times 10^{-5}$ & 0.0913 & 0.017 & 5.00 \\
& PsMeas & $2.7893 \times 10^{-5}$ & 0.0918 & 0.019 & 5.00 \\
& PsInj & $2.8894 \times 10^{-5}$ & 0.0842 & 0.016 & 5.00 \\
\hline
\end{tabular}

Table 8. State estimation results for the IEEE 14 test system

\begin{tabular}{cccccc}
\hline System & SE & $\sigma_{\Sigma}^{2}$ & $\xi$ & Time [s] & Iter. \\
\hline \multirow{6}{*}{ Classical } & $5.7825 \times 10^{3}$ & 1.0606 & 0.039 & 4.96 \\
& HCSE & $6.6138 \times 10^{5}$ & 0.0312 & 0.104 & 4.26 \\
IEE & RectI & $6.6136 \times 10^{5}$ & 0.0311 & 0.082 & 4.86 \\
30 & PsVolt & $6.7500 \times 10^{5}$ & 0.1538 & 0.048 & 4.11 \\
& SAHSE & $5.9630 \times 10^{5}$ & 0.0530 & $0.108+0.028$ & $1+3.04$ \\
& PsFlow & $6.3043 \times 10^{5}$ & 0.0313 & 0.063 & 4.24 \\
& PsMeas & $6.3095 \times 10^{5}$ & 0.0313 & 0.065 & 4.26 \\
& PsInj & $1.0658 \times 10^{4}$ & 0.1462 & 0.045 & 4.68 \\
\hline
\end{tabular}

Table 9. State estimation results for the IEEE 14 test system

\begin{tabular}{cccccc}
\hline System & SE & $\sigma_{\Sigma}^{2}$ & $\xi$ & Time $[\mathrm{s}]$ & Iter. \\
\hline \multirow{4}{*}{ Classical } & $4.5457 \times 10^{-3}$ & 0.9731 & 0.104 & 5.00 \\
& HCSE & $8.7830 \times 10^{-5}$ & 0.0407 & 0.420 & 4.99 \\
IEE & RectI & $8.7929 \times 10^{-5}$ & 0.0403 & 0.159 & 5.00 \\
57 & PsVolt & $6.5636 \times 10^{-5}$ & 0.0795 & 0.122 & 4.26 \\
& SAHSE & $5.6038 \times 10^{-5}$ & 0.0358 & $0.305+0.067$ & $1+3.00$ \\
& PsFlow & $1.0407 \times 10^{-4}$ & 0.0486 & 0.150 & 4.96 \\
& PsMeas & $1.0619 \times 10^{-4}$ & 0.0512 & 0.162 & 4.99 \\
& PsInj & $1.1417 \times 10^{-4}$ & 0.1061 & 0.128 & 4.75 \\
\hline
\end{tabular}

\subsection{The system state transition}

The time period of several minutes was considered, reflecting a real case scenario, in which the state estimator reruns every few minutes and the synchrophasors are coming into the control room much more frequently than the SCADA measurements. The time period was divided into $k_{\max }=25$ time-sample subintervals and in each of these subintervals the values of loads on certain buses were increased following a linear trend of 5\%,10\%, $20 \%$ or $30 \%$ over the whole period, with a random fluctuation of $3 \%$ in value. To obtain the set of measurements that capture the transition of the operating point due to the smooth loads change, the power flow calculation was run for each subinterval. The overload of the slack bus was avoided by changing the generator outputs according to the assignment of the participation factors.

The initialization necessary for the Holt's method was carried out using the voltage magnitudes and angles of the first two samples obtained from the power flow calculation. The parameters were set to $\alpha=0.8$ and $\beta=0.5$ as suggested in [15]. The diagonal elements of the matrix
$Q$ were kept constant at $10^{-6}$ during the whole simulation period, while the diagonal elements of the matrix $P_{0}$ were initialized to the same value due to the assumption of the high accuracy of the initial states and then they were updated [21].

The synchrophasors in each subinterval $k$ were inputs into the preprocessor that uses them to update the state estimate from its previous run in the subinterval $k-1$ and forwards the result to be used in the subinterval $k+1$. The state estimation obtained in the last run of the preprocessor at the end of the simulating period was taken into a new measurement vector together with the SCADA measurements and the WLS state estimator provided the state estimation of the complete power system.

To make the simulation more realistic, for the developed hybrid model and for other state estimators, the conventional measurements were taken randomly throughout the whole period of simulation, since they are not time synchronized; they are arriving in the control room randomly delayed. Also, the state estimators, with which the proposed estimator was compared, were run at the end of the simulating period, using the latest set of synchrophasors.

\section{RESULTS}

In this section the results gained from the simulations described in the previous section are given. Tables 7-9 provide the results of the proposed solution in comparison with the classical state estimator and other hybrid models.

The comparison of the results of the classical state estimator with the results obtained by applying the hybrid models reveals that the inclusion of the synchrophasors in the set of measurements does not contribute much to the speed and convergence of the calculation, but the estimation accuracy and the filtering of the measurement errors is significantly improved. It can be concluded that the SAHSE is comparable in view of the filtering of the measurement errors with the other methods, whereas the accuracy of the state estimation is improved. To distinguish the preprocessing of the synchrophasors from the subsequent iterative procedure, the number of iterations and the computation time for the SAHSE are given in two parts. The first part refers to the preprocessing of the synchrophasors which is taken as a single iteration, while the second part refers to the iterative WLS procedure. The computation time of the proposed solution is within acceptable limits, with the lowest number of iterations.

In the figures that follow, the results of the classical state estimator were discarded because the high values of its errors would make the results of the hybrid state estimators negligible. Figures 4 and 5 give the estimation errors of voltage angles and magnitudes for the IEEE 14 bus system. The errors of calculated active and reactive power flows for the IEEE 14 bus system are given in Figs. 6 and 7, respectively. For the IEEE test systems with 30 

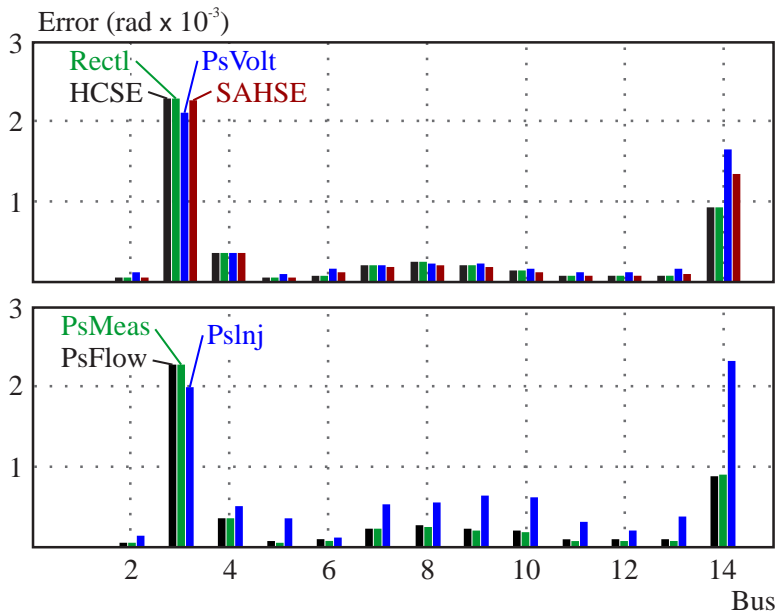

Fig. 4. Voltage angle errors for the IEEE 14 test system

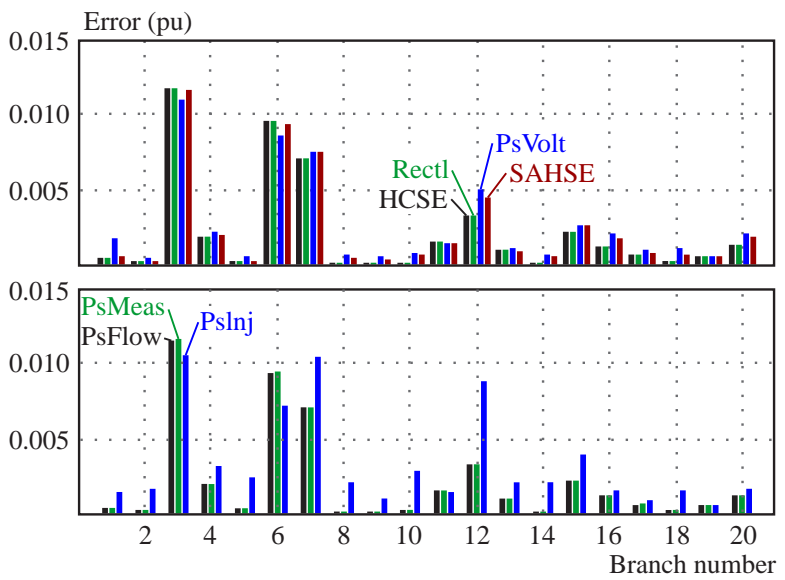

Fig. 6. Active power flow errors for the IEEE 14 test system

and 57 buses the results are not visualized due to space limitation and the larger number of buses and branches that would make figures unreadable.

\section{CONCLUSIONS}

The Synchrophasor Assisted Hybrid State Estimator presents the authors' proposition for the optimal inclusion of the synchrophasors into the set of measurements, with the focus on the enhancement of the state estimation accuracy. In the preprocessor, which uses the synchrophasors only, the state estimation for the part of the system observable by the PMUs is obtained. As the system state propagates in time from one operating point to another, the recursive algorithm is applied to take advantage of the consecutive sets of the synchrophasors. Without any transformation that would result in the loss of accuracy due to the propagation of measurement uncertainties, the result is used directly in the subsequent iterative procedure together with the conventional measurements. The flexible architecture of the given solution allows different operating options that take into account the possible unavailability of the synchrophasors as well as the increase
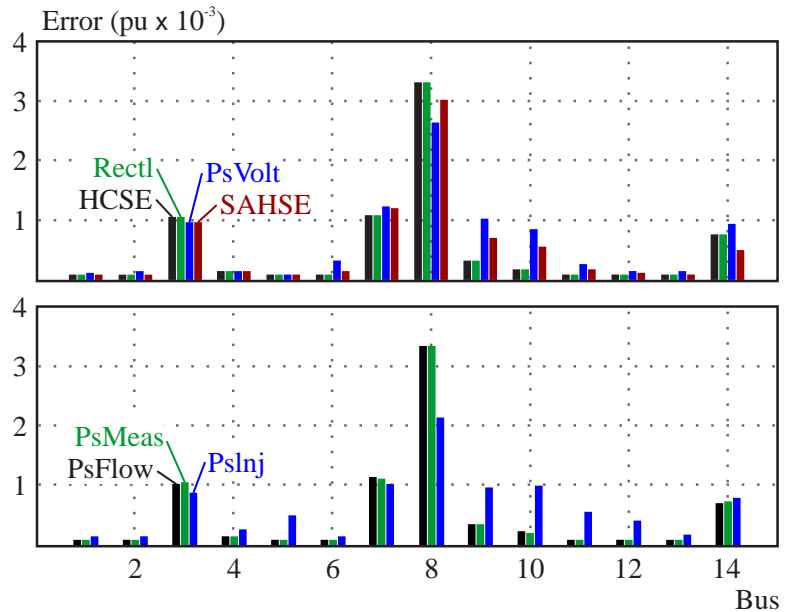

Fig. 5. Voltage magnitude errors for the IEEE 14 test system

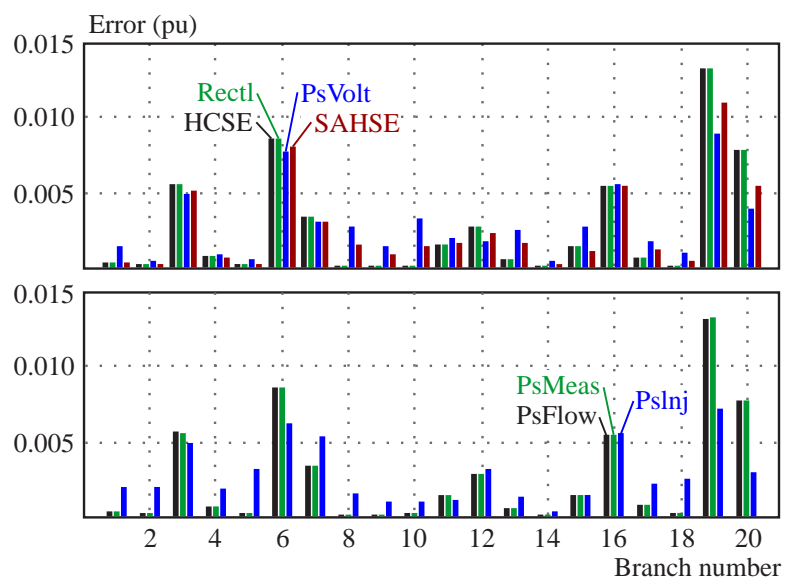

Fig. 7. Reactive power flow errors for the IEEE 14 test system

of the number of PMUs deployed in the power system that would have a positive effect on the estimator performance in general.

In order to validate the proposed methodology, the classical state estimator and the other hybrid state estimators were used. The state estimators were applied on the IEEE test systems with 14,30 and 57 buses and the results were obtained by carrying out 100 Monte Carlo analysis. To make the tests more realistic and to explore the benefits of using the multiple sets of the synchrophasors, in the case studies, a smooth transition of the system operating point in a minute range was assumed by simulating a linear increase of the loads on the selected buses. The performance metrics were used to evaluate the state estimation accuracy and filtering of the measurement errors as well as the speed and convergence of the calculation.

The results presented indicate that the proposed solution is comparable with the other hybrid models in view of filtering of the measurement errors. Although the total computation time of the proposed methodology is slightly larger due to preprocessing of the multiple sets of synchrophasors in comparison with only one set used in the other hybrid models, it is still within acceptable limits, 
which is confirmed with the smallest number of iterations necessary to reach the chosen tolerance. The accuracy of the developed model is the highest amongst the compared state estimators for all the test systems, which is crucial for the subsequent applications in the control room that use state estimator outputs. The enhancement of the state estimator accuracy supports the power system operator to challenge the issues associated with operating the power system in a secure, reliable and economic manner.

\section{REFERENCES}

[1] TERZIJA, V.-VALVERDE, G.-CAI, D.-REGUlSKI, P.MADANI, V.-FITCH, J.-SKOK, S.-BEGOVIC, M.PHADKE, A.: Wide Area Monitoring. Protection and Control of Future Electric Power Networks, Proceedings of IEEE 99 (2011), 80-93.

[2] CHAKRABARTI, S.-KYRIAKIDES, E.-TIANSHU, B.CAI, D.-TERZIJA, V. : Measurements Get Togetherjour IEEE Power and Energy Magazine.

[3] ABUR, A.-GOMEZ-EXPOSITO, A.: Power System State Estimation: Theory and Implementation, Marcel Dekker, New York, 2004

[4] MONTICELLI, A.: State Estimation in Electric Power Systems: A Generalized Approach, Kluwer Academic Publishers, Massachusetts, 1999.

[5] PHADKE, A.-THORP, J.: Synchronized Phasor Measurements and their Applications, Springer, 2008.

[6] Metrics for Determining the Impact of Phasor Measurements on Power System State Estimation, KEMA, Arnhem, The Netherlands, Mar 2006.

[7] BI, T. S.-QIN, X. H.-YANG, Q. X.: A Novel Hybrid State Etimator for Including Synchronized Phasor Measurements, Electric Power Systems Research 78 (2008), 1343-1352.

[8] VALVERDE, G.-CHAKRABARTI, S.-KYRIAKIDES, E.TERZIJA, V.: A Constrained Formulation for Hybrid State Estimation, IEEE Transactions on Power Systems 26 (2011), 1102-1109.

[9] CHAKRABARTI, S.-KYRIAKIDES, E.-VALVERDE, G.TERZIJA, V.: State Estimation Including Synchronized Measurements, Proceedings of the IEEE Power Tech conference, Bucharest, Romania, 2009, pp. 1-5.

[10] KIRINCIC, V.-SKOK, S.-TERZIJA, V. : A Hybrid State Estimator with Pseudo-Flows and Pseudo-Injections, International Review on Modelling and Simulations 6 (2013), 218-226.

[11] KIRINCIC, V.-SKOK, S.-MARUSIC, A.: A Hybrid Constrained State Estimator with Pseudo Injection Measurements, Przegld Elektrotechniczny 89 (2013), 137-142.

[12] ASPROU, M.-KYRIAKIDES, E.: Enhancement of Hybrid State Estimation using Pseudo Flow Measurements, Proceedings of Power and Energy Society General Meeting, Detriot, USA, 2011, pp. 1-7.

[13] MANOUSAKIS, N. M.-KORRES, G. N.-GEORGILAKIS, P. S. : Taxonomy of PMU Placement Methodologies, IEEE Transactions on Power Systems 27 (2012), 1070-1077.

[14] CHAKRABARTI, S.-KYRIAKIDES, E.-ALBU, M. : Uncertainty in Power System State Variables Obtained Through Synchronized Measurements, IEEE Transactions on Instrumentation and Measurement 58 (2009), 2452-2458.

[15] ISO-IEC-OIML-BIPM: Guide to the Expression of Uncertainty in Measurement, 1992.
16] VALVERDE, G.-TERZIJA, V.: Unscented Kalman Filter for Power System Dynamic State Estimation, IET Generation, Transmission \& Distribution 5 (2011), 29-37.

17] Leite da Silva, A. M.-DO COUTTO FILHO, M. B.- de QUEIROZ, J. F. : State Forecasting in Electric Power Systems, IEE Proceedings on Generation, Transmission and Distribution 130 (1983), 237-244.

18] CHRISTIE, R. : Power System Test Archive (Aug 1999), [Online, 22/12/2012], http://www.ee.washington.edu/research/pstca.

[19] Al-OThMAN, A. K.-IRVING, M. R.: Uncertainty Modeling in Power System State Estimation, IET Generation, Transmission \& Distribution 152 (2005), 233-239.

[20]] Model 1133A GPS-Synchronized Power Quality/ Revenue Standard, Operation Manual, Arbiter Systems, Inc. CA, USA.

[21] CHUN-LIEN, S.-CHAN-NAN, L.: Interconnected Network State Estimation using Randomly Delayed Measurements, IEEE Transactions on Power Systems 26 (2001), 870-878.

Received 1 July 2015

Vedran Kirincic $(\mathrm{PhD})$ received the MSc degree in electrical engineering from the Faculty of Engineering, University of Rijeka, Rijeka, Croatia in 2007 and the $\mathrm{PhD}$ degree in electrical engineering from the Faculty of Electrical Engineering and Computing, University of Zagreb, Zagreb, Croatia in 2013. He has been with the Department of Power Systems of the Faculty of Engineering, University of Rijeka, since 2009, as a senior research assistant. He was a visiting researcher at University of Manchester, U.K., in spring 2012 and at the KIOS Research Center for Intelligent Systems and Networks, University of Cyprus, Cyprus, in spring 2014. His research interests include state estimation and synchronized phasor measurements in power systems.

Srdjan Skok $(\mathrm{PhD})$ received the BSc degree in electric power engineering from the Faculty of Electrical Engineering and Computing, University of Zagreb, Zagreb, Croatia in 1995, and the MSc and PhD degree in electrical engineering from the same University in 2000 and 2004, respectively. He has been with the Department of Power Systems of the Faculty of Engineering, University of Rijeka, since 2008, as an associate professor. His field of research and teaching interest is related to power system protection and control as well as synchronized measurement technologies and smart grid implementation into power transmission grid. He has very successful collaboration with subjects from industry on many expert studies.

Dubravko Frankovic $(\mathrm{PhD})$ received the BSc degree in electric power engineering from the Faculty of Electrical Engineering and Computing, University of Zagreb, Zagreb, Croatia in 1999, and was granted MSc and PhD degree in electrical engineering from the same University in 2003 and 2009, respectively. He has been with the Department of Power Systems of the Faculty of Engineering, University of Rijeka, since 2001, as a junior researcher. Currently, he is the assistant professor in power system engineering at the Faculty of Engineering, University of Rijeka, Croatia and the head of the Department of Power Systems of the Faculty of Engineering, University of Rijeka. His major fields of interests are transmission and distribution power systems, energy efficiency and renewable energy sources implementation. 